\title{
Segmentation of Tumor Region from Brain Mri Images Using Fuzzy C-Means Clustering And Seeded Region Growing
}

\author{
Harsimranjot Kaur ${ }^{1}$, Dr. Reecha Sharma ${ }^{2}$ \\ ${ }^{I}$ (M. Tech Student, Department of ECE, Punjabi University, Patiala, India) \\ ${ }^{2}$ (Assistant Professor, Department of ECE, Punjabi University, Patiala, India)
}

\begin{abstract}
The detection of brain tumor is one of the most challenging tasks in the field of medical image processing, since brain images are very complicated and tumors can be analyzed efficiently only by the expert radiologists. Therefore, there is a significant need to automate this process. In this paper, a method for the automatic detection of the tumor from the brain magnetic resonance imaging (MRI) images has been proposed. For this, the region-based segmentation of the input MRI image is done. The wavelet-based decomposition of the input image is done and the input image is reconstructed on the basis of soft thresholding for the enhancement of the image. After that, fuzzy c-means clustering (FCM) followed by seeded region growing is applied to detect and segment the tumor from the brain MRI image and finally comparison with Sobel operator is done on the basis of Relative Ultimate Measurement Accuracy (RUMA) and Standard Deviation (SD). The results for the proposed technique are better than the results obtained by using Sobel operator.
\end{abstract}

Keywords: Brain tumor segmentation, FCM, Region growing, Wavelet decomposition.

\section{Introduction}

The brain tumor is a very common disease now-a-days which has devastated many lives and the count is still increasing. In the field of biomedical imaging, the segmentation of brain tumor from the MRI images has become an emergent area of research. The magnetic resonance imaging (MRI) scans produce detailed images of the internal structure of human brain and other parts of the body. The MRI scan is preferred over other techniques like computed tomography (CT) scan or ultrasound for brain tumor diagnosis as it provides more clear images of those parts of brain also which cannot be seen with other techniques. The accurate detection of the size and location of the tumor in the human brain plays a significant role in the diagnosis of the tumor. The segmentation of brain tumor from MRI scan is done manually by experts. The manual segmentation of brain tumor region is a very difficult and time-consuming task and the accuracy of the result depends on the experience of the physician. In this work, a method for automated detection of brain tumor from MRI scan is being presented. The system for automated detection of the brain tumor from the MRI helps in the extraction of region of interest from the MRI scans which of great significance to the physicians in the diagnostic and treatment process. Then the comparison of the proposed method is done with the Sobel operator and the performance analysis is done on the basis of accuracy, sensitivity, specificity and precision values.

\section{Related Work}

The segmentation of brain tumor from MRI images is a major topic of biomedical research and extensive work had been done in this area. A large number of different techniques are available for this purpose [1] [2]. The researchers have used edge based segmentation techniques, thresholding and region based segmentation techniques [3], fuzzy clustering and neuro-fuzzy techniques and have been able to segment tumor region [4] [5]. Asra Aslam et al. presented an improved edge detection algorithm for segmentation of brain tumor [6]. This algorithm is based on Sobel edge detection method. The brain tumors extracted from proposed method using Sobel method along with closed contour algorithm are better than the tumors detected using Sobel method only. Roy et al. proposed a completely automatic algorithm to detect tumors by utilizing symmetry analysis [7]. It produces great results in images with non-uniform intensity distributions also. S. Roy et al. explored a technique to distinguish tumor in brain MRI images [8]. It applied segmentation technique on the MRI image of brain tumor, converting it into binary image and then applied morphological operations on it. The results obtained by this technique were efficient. Malakooti et al. proposed a tumor segmentation technique which uses combined fuzzy logic and neural networks and extracts the boundary taking into account level set method [9]. The proposed technique gave better results as compared to other existing techniques. Deshmukh et al. presented an automated recognition system for the MRI image using the neuro-fuzzy logic [10]. It takes the idea that manual analysis of brain tumor is time annihilating and produces inaccurate results. This proposed method is quick in execution, proficient in arrangement and simple in implementation. In the proposed work segmentation of the input and performance analysis is done on the basis of accuracy, sensitivity, specificity and precision values. 


\section{Proposed technique for brain tumor detection from MRI}

In this work, the basic concept is to detect the tumor region in brain MRI. For this, the region-based segmentation of the input image is done. The proposed work can be useful for improved brain tumor detection. The proposed work uses wavelet decomposition for image smoothing. After that fuzzy c-means clustering is implemented to cluster the input image into a number of clusters. This step puts the brain tumor into a significant cluster. Therefore it becomes easy to process only that region which contains the tumor. Then connected component analysis and seeded region growing are applied to find the tumor region. The flowchart for the procedure is as given in fig. 1 .

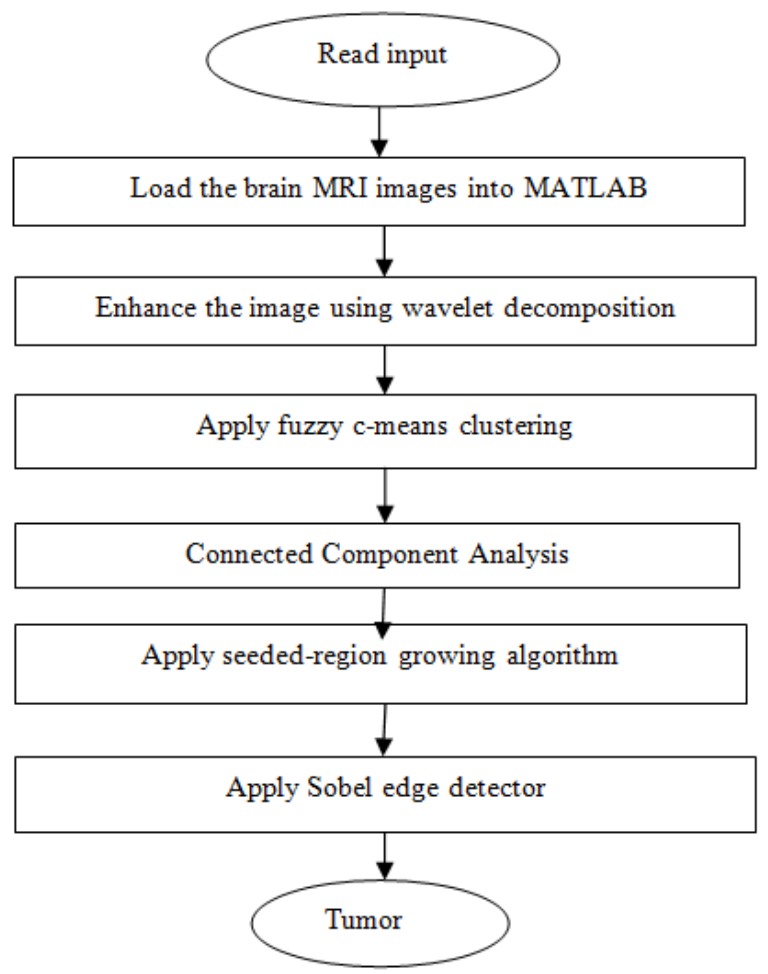

Fig.1: Flowchart for brain tumor extraction

\section{Step 1: Input the MRI image into MATLAB}

Load the brain MRI image into MATLAB and convert it to grayscale if it is RGB colored image. The input brain tumor image is shown in fig. 2 .

\section{Step 2: Apply Wavelet Decomposition}

Apply wavelet based decomposition for smoothing and de-noising the image. In the proposed work, 'bior 3.5' wavelet has been used to decompose the image up to four levels. The approximation and the detailed horizontal, vertical and diagonal coefficients of the image are extracted. Soft thresholding is applied before reconstruction of the decomposed image. The de-noised image is shown in fig. 3.

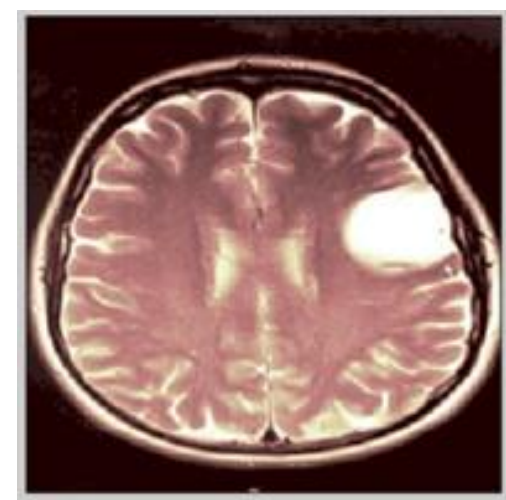

Fig.2: Input brain tumor image

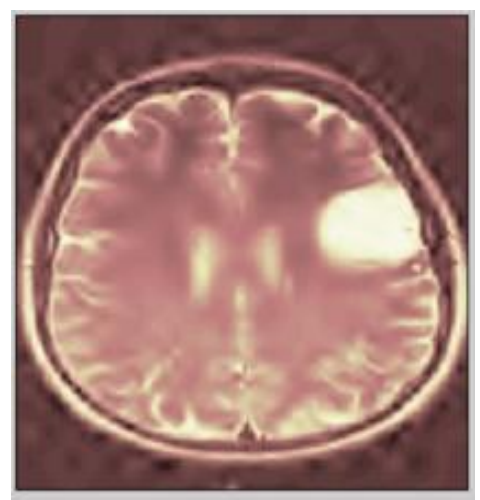

Fig.3: De-noised image using wavelets 


\section{Step 3: Apply fuzzy c-means clustering}

Apply fuzzy c-means clustering (FCM) to get segmentation of image into four clusters. FCM clustering is an unsupervised method for the analysis of given input image. The fcm clustering algorithm assigns membership functions to every pixel in an image corresponding to each cluster center based on the distance of the cluster center from that pixel [11]. The pixels near to the cluster center have higher membership function towards that particular cluster. The FCM clustered image is shown in fig. 4.

\section{Step 4: Connected component analysis}

The connected component analysis of the output of fuzzy clustering is done. The pixels of the binary image obtained after applying FCM are labeled as 0 are the background object pixels. The pixels which are labeled as 1 make up one object; the pixels being labeled as 2 make up a second object and so on. In this paper, we used area and perimeter property of the regions and used them to eradicate unwanted objects in the binary image and found the object which contains maximum pixels of brain tumor in it. Further, the centroid of the final object is used as a seed point to get segmentation of tumor using region growing algorithm. The binary image is shown in fig. 5.

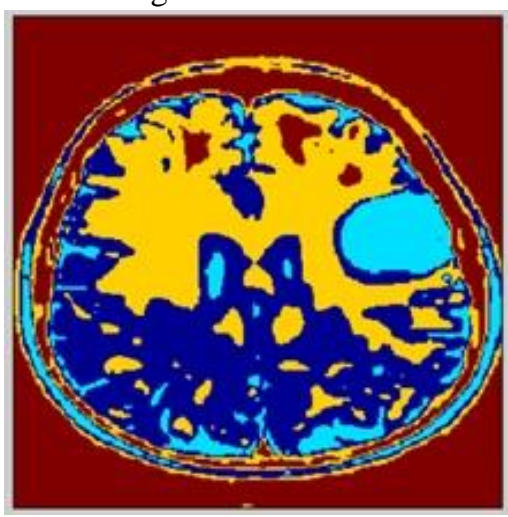

Fig.4: FCM clustered image

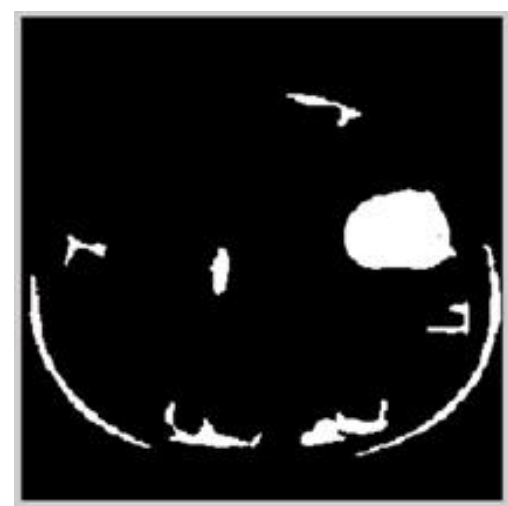

Fig.5: Objects in image after connected component analysis

\section{Step 5: Region growing algorithm for segmenting out tumor}

The basic idea behind the technique of region growing is grouping the pixels with similar properties together to form a particular region. The seed point for the final object left after connected component analysis is calculated automatically and the neighboring pixels around the seed point pixels are analyzed by the predetermined region growing formula. When all the neighboring pixels are included into the seed pixels domain, at that point the region is said to be grown and region growing stops. The region growing results are shown in fig. 6.

\section{Step 6: Edge detection of tumor}

After the region growing algorithm, edge detection of the tumor region is done using the Sobel operator. The detected boundary of tumor is shown in fig. 7.

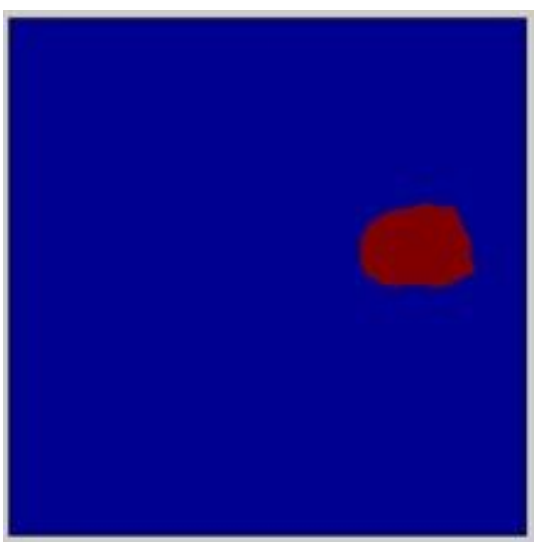

Fig.6: Tumor detected after region growing algorithm

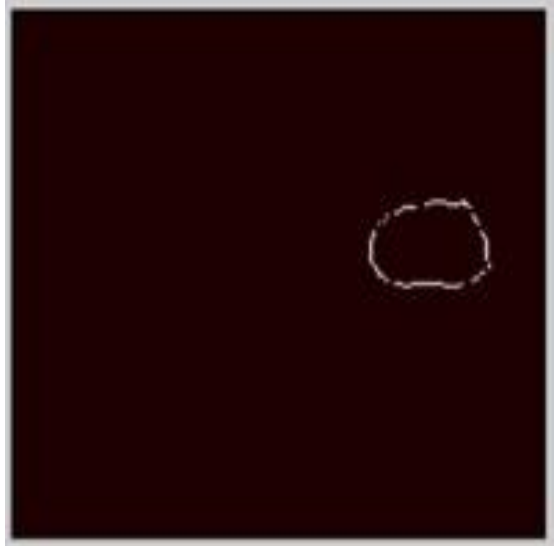

Fig.7: Tumor edge detection using 'Sobel' filter 


\section{Experimental results and discussions}

The results thus obtained by the proposed method are analyzed using the performance parameters [12]. The parameters being calculated include Relative Ultimate Measurement Accuracy (RUMA) and Standard Deviation (SD). The lesser the values of Relative Ultimate Measurement Accuracy (RUMA) and Standard Deviation (SD), the better is the performance of the system. The ground truth image is taken as the reference image for comparison.

1. Relative Ultimate Measurement Accuracy (RUMA): The Relative Ultimate Measurement Accuracy is calculated for the objects which have been segmented. It is computed based on the values of the features extracted from the segmented image being compared to that of the reference image. For the better performance of the segmentation algorithm, the lower value of RUMA is required. The mathematical expression for RUMA can be described as:

$$
\text { RUMA }=\frac{\left|R_{f}-S_{f}\right|}{R_{f}} \times 100
$$

where, $R_{f}$ is the feature value obtained from the reference image and $S_{f}$ is the feature value obtained from the segmented image.

2. Standard Deviation (SD): It is a measure of the variation in the delineated regions. It shows the extent to which the values of segmented region vary from the mean value. The lower values of SD means there is less variability in the extracted regions and hence the better is the performance of the system. It is the square root of the variance. The mathematical expression for SD can be defined as:

$$
\mathrm{SD}=\left[\frac{1}{\mathrm{~N}-1} \sum_{\mathrm{f}=1}^{\mathrm{N}}\left|\mathrm{S}_{\mathrm{f}}-\mu\right|^{2}\right]^{\frac{1}{2}}
$$

where, $\mu$ is the mean of the values of segmented image $\left(\mathrm{S}_{\mathrm{f}}\right)$

$$
\mu=\frac{1}{\mathrm{~N}} \sum_{\mathrm{f}=1}^{\mathrm{N}} \mathrm{S}_{\mathrm{f}}
$$

For performance analysis, the values obtained by using the proposed algorithm are being compared with the

\begin{tabular}{|c|c|c|c|c|c|}
\hline \multirow[b]{2}{*}{ S.NO. } & \multirow{2}{*}{$\begin{array}{l}\text { INPUT BRAIN MRI } \\
\text { IMAGE }\end{array}$} & \multicolumn{2}{|c|}{$\begin{array}{c}\text { RUMA } \\
\text { (RELATIVE ULTIMATE } \\
\text { MEASUREMENT ACCURACY) }\end{array}$} & \multicolumn{2}{|c|}{$\begin{array}{c}\text { SD } \\
\text { (STANDARD DEVIATION) }\end{array}$} \\
\hline & & $\begin{array}{l}\text { PROPOSED } \\
\text { METHOD }\end{array}$ & \begin{tabular}{|l|} 
SOBEL \\
OPERATOR
\end{tabular} & $\begin{array}{l}\text { PROPOSED } \\
\text { METHOD }\end{array}$ & $\begin{array}{l}\text { SOBEL } \\
\text { OPERATOR }\end{array}$ \\
\hline 1. & & 11.2210 & 45.1227 & 0.1953 & 0.2067 \\
\hline 2. & & 58.6592 & 90.9961 & 0.0886 & 0.1369 \\
\hline 3. & & 71.4488 & 90.6805 & 0.0783 & 0.1453 \\
\hline 4. & & 21.0181 & 73.6969 & 0.1528 & 0.1713 \\
\hline 5. & & 25.9738 & 38.1092 & 0.2936 & 0.3644 \\
\hline 6. & & 27.6790 & 82.2806 & 0.1574 & 0.1842 \\
\hline
\end{tabular}
values being obtained by using Sobel operator. The values are given in Table 1 as given below:

Table 1: The performance parameter values computed for various brain MRI images 


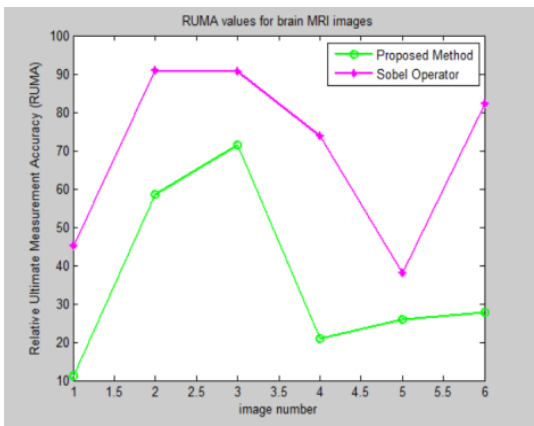

(a)

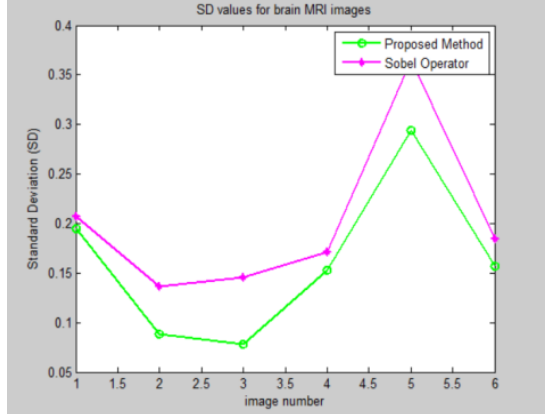

(b)

Fig. 8: Performance comparison in terms of (a) Relative Ultimate Measurement Accuracy (RUMA); (b) Standard Deviation (SD).

From the outcomes appeared above it has been found that the proposed algorithm has high Relative Ultimate Measurement Accuracy (RUMA) and Standard Deviation (SD) values than those obtained by using the Sobel operator. The results for a few pictures have been shown as depiction while we attempted the calculation on a number of distinct images.

\section{Conclusion}

In this work, a method which can help the radiologists in the automatic detection of the brain tumor from MRI images has been proposed. It is observed that the results obtained by the proposed method are better than those obtained by applying Sobel operator as they have lower values of RUMA and SD which implies better performance. The proposed method works well on different types of brain tumor images and gives better results for the input MRI images than Sobel operator.

\section{Future scope}

This algorithm shows good results on the basis of performance parameters. However, it does not classify the brain tumor on the basis of its type. In future, dataset of brain MRI images with different stages of tumor can be collected and based on which a good classification system can be designed. So, in future, data collection and classification are the significant steps which need to be carried out so that a fully automated brain tumor diagnosis system can be designed.

\section{References}

[1]. Aswathy, S. U., G. Glan Deva Dhas, and S. S. Kumar, "A survey on detection of brain tumor from MRI brain images", In Control, Instrumentation, Communication and Computational Technologies (ICCICCT), International Conference on, pp. 871-877, IEEE, 2014.

[2]. Gordillo, Nelly, Eduard Montseny, and Pilar Sobrevilla, "State of the art survey on MRI brain tumor segmentation", Magnetic resonance imaging, Elsevier, vol. 31, no. 8, pp. 1426-1438, 2013.

[3]. Viji, K.A. and JayaKumari, J., "Modified texture based region growing segmentation of MR brain images" In Information \& Communication Technologies (ICT), IEEE Conference on, pp. 691-695, IEEE, April 2013.

[4]. Ahmed, M.N., Yamany, S.M., Mohamed, N., Farag, A.A. and Moriarty, T. "A modified fuzzy c-means algorithm for bias field estimation and segmentation of MRI data", IEEE transactions on medical imaging, vol. 21, no. 3, pp.193-199, IEEE, 2002.

[5]. Aswathy, S. U., G. Glan Deva Dhas, and S. S. Kumar, "A survey on detection of brain tumor from MRI brain images", In Control, Instrumentation, Communication and Computational Technologies (ICCICCT), International Conference on, pp. 871-877, IEEE, 2014.

[6]. Aslam, Asra, Ekram Khan, and MM Sufyan Beg, "Improved Edge Detection Algorithm for Brain Tumor Segmentation", Procedia Computer Science, vol. 58, pp. 430-437, 2015.

[7]. Roy, Sudipta, and Samir K. Bandyopadhyay, "Detection and Quantification of Brain Tumor from MRI of Brain and its Symmetric Analysis", International Journal of Information and Communication Technology Research, vol. 2, no. 6, 2012.

[8]. Roy, Sudipta, "Brain Tumor segmentation and quantification from MRI of brain", Journal of Global Research in Computer Science, vol. 2, no. 4: pp. 155-159, 2011.

[9]. M.V. Malakooti, S. A. Mousavi and N. H. Taba, "MRI brain Image Segmentation Using Combined Fuzzy Logic and Neural Networks for the Tumor Detection", Journal of Academic and Applied Studies, vol. 3, no. 5, 2013.

[10]. Deshmukh, R. J., and R. S. Khule, "Brain tumor detection using artificial neural network fuzzy inference system (ANFIS)", International Journal of Computer Applications Technology and Research vol. 3, no. 3, pp. 150-154, 2014.

[11]. Shen, Shan, William Sandham, Malcolm Granat, and Annette Sterr, "MRI fuzzy segmentation of brain tissue using neighborhood attraction with neural-network optimization", IEEE transactions on information technology in biomedicine, vol. 9, no. 3, pp. 459467, 2005.

[12]. Afina S. Glas et al, "The diagnostic odds ratio: a single indicator of test performance", Journal of Clinical Epidemiology, vol. 56, pp. 1129-1135, 2003. 